\title{
Estudio ab initio de la capacidad antioxidante de una familia de alcaloides imidazólicos versus flavonoides
}

\author{
Ab initio study of the antioxidant capacity of an imidazole alkaloids \\ versus flavonoids family
}

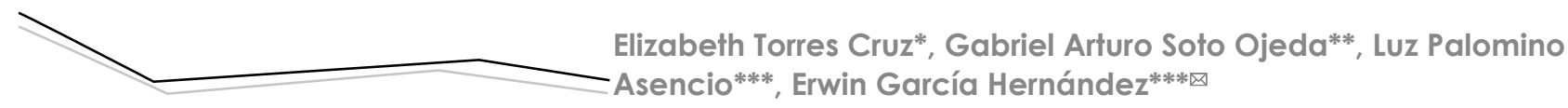

Torres Cruz, E., Soto Ojeda, G. A., Palomino Asencio, L., \& García Hernández, E. (2017). Estudio ab initio de la capacidad antioxidante de una familia de alcaloides imidazólicos versus flavonoides. Investigación y Ciencia de la Universidad Autónoma de Aguascalientes, 25(72), 30-35.

RESUMEN

En este trabajo se estudiaron tres alcaloides imidazólicos; Lepidina D, E y F, y tres flavonoides; crisina, quercetina y camferol dentro del marco de la Teoría de Funcionales de la Densidad para determinar su capacidad antioxidante mediante el mecanismo de transferencia electrónica. Se aplicó la metodología B3LYP/6-31+G(d) para obtener las estructuras de mínima energía y los índices de reactividad globales. Para los alcaloides y flavonoides no hubo cambios sustanciales en los parámetros geométricos. En el caso de los índices de reactividad globales, el Potencial de lonización muestra que en los alcaloides, la Lepidina $\mathrm{F}$ es el que tiene el valor más pequeño, sugiriéndolo como buen antioxidante con respecto

Palabras clave: antioxidantes; ab initio; alcaloides; flavonoides; Teoría de Funcionales de la Densidad; índices de reactividad globales.

Keywords: antioxidants; ab initio; alkaloids; flavonoids; Density Functional Theory; global reactivity predictors.

\section{Recibido: 23 de marzo de 2017, aceptado: 1 de septiembre de 2017}

* División de Biología, Instituto Tecnológico Superior de Zacapoaxtla. Carretera Acuaco-Zacapoaxtla km 8, Col. Totoltepec, C. P. 73680, Zacapoaxtla, Puebla, México. Correo electrónico: marelyz_tc97@ hotmail.com

** Facultad de Química Farmacéutica Biológica, Universidad Veracruzana. Circuito Gonzalo Aguirre Beltrán s/n, Zona Universitaria, C. P. 91000 Xalapa, Veracruz, México. Correo electrónico: gsoto@uv.mx

*** Departamento de Posgrado e Investigación, División de Mecatrónica Instituto Tecnológico Superior de Zacapoaxtla. Carretera AcuacoZacapoaxtla km 8, Col. Totoltepec, C. P. 73680, Zacapoaxtla, Puebla, México. Correo electrónico: palominoluz21@gmail.com; erwin.garcia@ live.itsz.edu.mx

$凶$ Autor para correspondencia a los flavonoides. Una conclusión importante de este trabajo es que los alcaloides podrían fungir como buenos antioxidantes debido a la fracción imidazol.

\section{ABSTRACT}

A Density Functional Theory study of three imidazole alkaloids; Lepidine D, E and F, and three flavonoids; chrysin, quercetin and kaempferol was carried out to obtain information about its antioxidant capacity. The B3LYP/6-31+G(d) method was applied to find the minimum in the potential energy surface and the global reactivity predictors. There was not observed substantial changes in the structural parameters in both families. By the side of reactivity predictors, the Ionization Potential suggests that alkaloids are good antioxidants versus flavonoids, specially the Lepidine $\mathrm{F}$, which present the smallest value of this quantity. An important conclusion in this work is the participation of the imidazole group, suggesting that alkaloids are good antioxidant due to presence of this moiety.

\section{INTRODUCCIÓN}

Los antioxidantes son compuestos bioactivos que juegan un papel primordial en la preservación de los alimentos y productos farmacéuticos al protegerlos del proceso de oxidación (Aksu, Topal, Gulcin, Tümer, \& Göksu, 2015; Shahidi \& Zhong, 2010; Zou \& Akoh, 2015), además de proteger al cuerpo humano de varias enfermedades cardiovasculares, cáncer y envejecimiento por la captura de radicales libres (Gülcin, Büyükokuroglu, Oktay, \& Küfrevioglu, 2003). Un antioxidante es un compuesto que previene o retarda la oxidación de sustratos aun si éste está 
disponible en concentraciones sustancialmente bajas con respecto a los sustratos oxidados (Gülcin, Büyükokuroglu, \& Küfrevioglu, 2003).

Ejemplo de antioxidantes naturales son los compuestos fenólicos o polifenoles, que constituyen uno de los grupos más numerosos y altamente distribuidos en el reino vegetal, con cientos de estructuras actualmente conocidas. La expresión compuestos fenólicos, se refiere a sustancias que contienen un arreglo que posee un anillo aromático de seis carbonos, sustituido con uno o más grupos hidroxilo $(-\mathrm{OH})$ (Halake, Birajdar, \& Lee, 2016; Villuendas-Rey, Alvarez-Idaboy, \& Galano, 2015). Dentro de estos, se encuentran los flavonoides, que en su mayoría contienen grupos fenoles en su estructura.

Los flavonoides son una familia de compuestos a los que se les atribuye gran cantidad de efectos biológicos y/o terapéuticos, como prevención de cáncer, diabetes, enfermedades neurológicas (Middleton, Kandaswami, \& Theoharides, 2000; Qian, Wang, Fan, Tan, \& Yang, 2015) y la más importante, como antioxidantes, donde bastos estudios han comprobado su gran efectividad como retardadores de estrés oxidativo y daño celular (Havsteen, 2002).

Por otro lado, se puede mencionar a los alcaloides imidazólicos como compuestos que también contienen en su estructura grupos fenólicos, algunos de los cuales fueron aislados por Maier, Gundlanch y Zenk (1998) de Lepidium sativum. Los alcaloides son compuestos orgánicos de origen natural, nitrogenados, heterocíclicos y generalmente se sintetizan a partir de aminoácidos; se les puede asociar con neurotoxicidad debido a la similitud con moléculas que participan en la transmisión de las señales del sistema nervioso, por lo que tienen la capacidad de bloquear neuro-receptores, intermediarios de la transducción de señal neuronal y canales iónicos de vertebrados e insectos (Sepúlveda-Jiménez, PortaDucoing, \& Rocha-Sosa, 2003). A pesar de esto, hay estudios donde relacionan a los alcaloides con antioxidantes (Srivastava, Tandon, Ayala, \& Jain, 2011), aspecto de gran interés para profundizar.

Los autores del presente trabajo no tienen conocimiento de un análisis teórico que evalúe la capacidad antioxidante de los alcaloides imidazólicos como la Lepidina D, E y F, por lo que en este artículo se presenta un estudio de estructura electrónica en el que se obtuvieron algunos índices de reacti- vidad globales como el potencial de ionización, los orbitales moleculares y la función de Fukui asociada a la donación de carga para los alcaloides antes mencionados y los flavonoides quercetina, crisina y camferol, con el fin de comparar sus características electrónicas y proponer a los alcaloides como buenos antioxidantes.

\section{MATERIALES Y MÉTODOS}

Para el presente estudio se tomaron en cuenta tres lepidinas aisladas por Maier y colaboradores (1998) provenientes de Lipidium sativum, las cuales comparten ciertas características estructurales, como puede apreciarse en la figura 1. Por parte de los flavonoides, se decidió estudiar tres estructuras que tuvieran grupos fenol, catecol y resorcinol. Inicialmente se generaron las primeras estructuras con una versión de prueba del programa Chemcraft v1.8 y se procedió a llevar a cabo una optimización de geometría de las estructuras estudiadas (figura 1) para obtener la conformación de más baja energía dentro del marco de la Teoría de Funcionales de la Densidad (DFT) con el método B3LYP (Becke, 1993), el cual es adecuado para tratar sistemas con fracciones fenólicas (OrtegaMoo, Garza, \& Vargas, 2016), acoplado a un conjunto base doble zeta con una función de polarización (d) para átomos pesados, 6-31+G(d) (Ditchfield, Hehre, \& Pople, 1971).

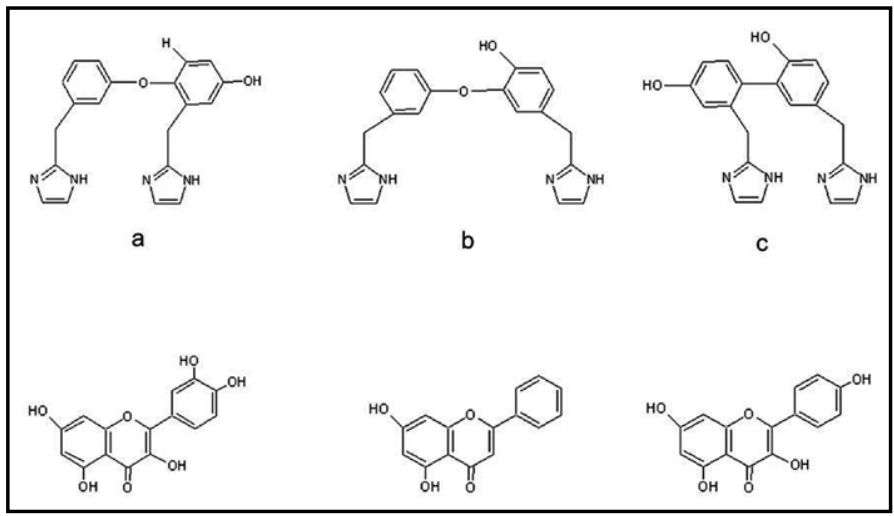

Figura 1. Estructuras de los alcaloides: a) Lepidina D, b) Lepidina E, c) Lepidina F y flavonoides, d) quercetina, e) crisina y f) camferol. Elaboración propia.

Se obtuvieron las frecuencias vibracionales para corroborar que las estructuras estuvieran en un mínimo de energía dentro de la superficie de energía potencial. Para verificar la capacidad antioxidante de los alcaloides y flavonoides, se tomó en cuenta el proceso mediante transferencia de carga (SET), 
en el cual se evalúa solamente la donación de un electrón o fracción del mismo. Para ello, lo primero que se hizo fue calcular el potencial de lonización vertical (PI) mediante el $\triangle S C F$ (o aproximación de diferencias finitas) usando la siguiente relación:

$$
P I=E_{(N-1)}-E_{(N)}
$$

Ec. 1

donde $E_{(N-1)}$ corresponde a la energía del sistema catiónico, y $E_{(N)}$ a la del sistema neutro. Además, se comparó con la obtenida mediante el eigenvalor del orbital molecular ocupado de más alta energía, HOMO. Para obtener información del sitio de la molécula donde se lleva a cabo la remoción del electrón se mapeó el orbital HOMO de todas las estructuras. Asimismo, debido a que en un proceso de transferencia de carga no siempre se donan electrones completos, se obtuvo la función de Fukui (Parr \& Yang, 1984) asociada al proceso de donación de carga ( $f^{-}$), la cual mide cómo cambia la densidad electrónica $[\partial \rho(r)]$ cuando pierden electrones $[\partial \mathrm{N}]$, manteniendo el potencial externo fijo $[v(r)]$; es decir, que los núcleos se mantienen fijos. Esta función se puede obtener mediante una diferencia de densidades entre el sistema neutro $\left[\rho_{N_{0}}(r)\right]$ menos el sistema catiónico [ $\rho_{N_{0}-1}(r)$ ], como lo muestra la siguiente ecuación:

$$
f^{-}=\left(\frac{\partial \rho(r)}{\partial N}\right)_{v(r)}^{-}=\rho_{N_{0}}(r)-\rho_{N_{0}-1}(r) \quad \text { EC. } 2
$$

El cálculo de los índices globales de reactividad se realizó utilizando el funcional B3LYP (Becke, 1993: 5648), un conjunto de base doble zeta con una función de polarización (d) para los átomos pesados y una función difusa (+), 6-31+G (d) (Ditchfield et al., 1971: 724). Todos los cálculos fueron realizados con el paquete computacional NWChem en su versión 6.3 (Valiev et al., 2010: 1477).

RESULTADOS

\section{Análisis conformacional}

En la figura 2 se muestran las estructuras optimizadas de los alcaloides y flavonoides con el método B3LYP/6-31+G(d).

Se evaluaron algunos parámetros geométricos entre el camferol, quercetina y crisina, en donde no se encontraron diferencias significativas en cuanto a la distancia de enlace y la amplitud de

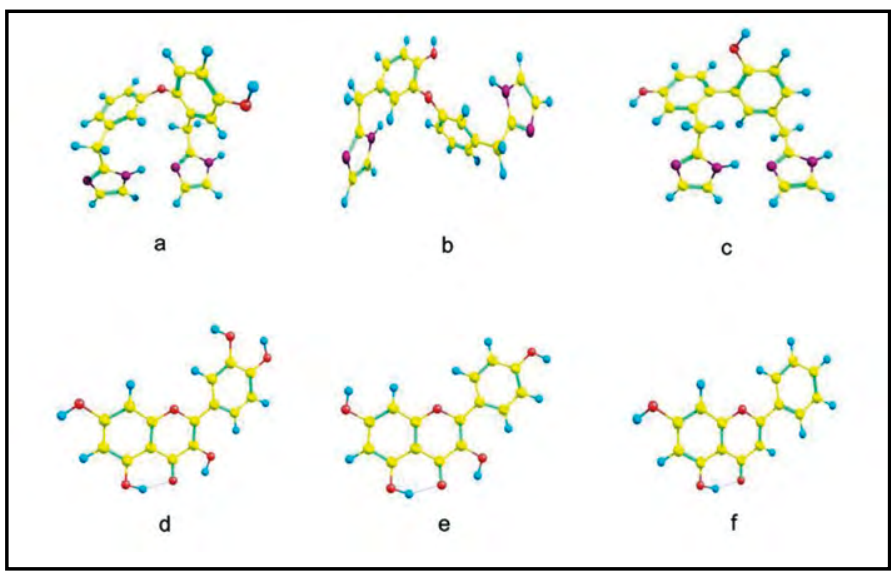

Figura 2. Estructuras optimizadas de: a) Lepidina D, b) Lepidina $E$, c) Lepidina $F$, d) quercetina, e) camferol y f) crisina con la metodología B3LYP/6-31+G(d).

Elaboración propia.

sus ángulos. De la figura 2 se puede observar que en todos los flavonoides en la fracción resorcinol se forma un puente de hidrógeno intramolecular (IHB) entre el $-\mathrm{OH}$ y el grupo carbonilo $(-\mathrm{CO})$ con una distancia de $1.7 \AA$, atribuyendo a la estructura mayor estabilidad; sin embargo, ya se ha reportado que en compuestos con fracción catecol y resorcinol el enlace intramolecular no afeca los índices globales de reactividad (García-Hernández \& Garza, 2017; Ortega-Moo et al., 2016: 177). De la misma manera, en la figura 2 ( $a, b$ y c) se muestran las geometrías más estables para los alcaloides Lepidina D, E y F, respectivamente. Al igual que en los flavonoides, en los alcaloides no se observan diferencias en parámetros geométricos, principalmente en las fracciones fenol e imidazol. El análisis de frecuencias vibracionales arrojó valores positivos en todos los casos.

\section{Reactividad química}

En esta sección del artículo se muestran los resultados de los índices de reactividad globales. La evaluación de capacidad antioxidante de las especies estudiadas se basó en el mecanismo SET. El primer índice evaluado en este trabajo fue el Pl. Para la obtención de este valor se usaron el $\triangle S C F$ (EC. 1) y la energía del orbital HOMO. En la tabla 1 se muestran los valores de Pl. De dicha tabla podemos rescatar que los valores obtenidos con la energía del $\mathrm{HOMO}$ están por debajo de los arrojados por el $\triangle S C F$ en $1.5 \pm 0.2 \mathrm{eV}$. Evidentemente, los números obtenidos mediante diferencias finitas son más confiables, ya que el eigenvalor del HOMO depende totalmente del funcional a utilizar y la corrección asintótica 


\section{InVESTIGACIÓn Y CIERCIA DE LA UחIVERSIDAD AUTÓNOMA DE AGUASCALIERTES}

del potencial de Kohn-Sham, por lo que el análisis se realizó mediante los valores del $\triangle \mathrm{SCF}$. Para los flavonoides se puede ver que el valor más bajo del $\mathrm{Pl}$ es el de la quercetina con $7.32 \mathrm{eV}$, indicando que es más fácil remover un electrón de ese sistema que de la crisina, el cual tiene un valor de $7.95 \mathrm{eV}$. Esto contribuye a las propuestas de la literatura donde se expone que los compuestos con grupos fenólicos son más susceptibles a la donación de electrones, por lo que su PI es más bajo con respecto a los que contienen grupos resorcinol (Ortega-Moo et al., 2016, p. 177).

Tabla 1

PI para los sistemas estudiados con B3LYP/6-3 I+G(d)

\begin{tabular}{lcc}
\hline Molécula & Energía HOMO & $\Delta$ SCF \\
\hline Lepidina F & 5.67 & 7.09 \\
Lepidina D & 5.64 & 7.28 \\
Lepidina E & 6.08 & 7.40 \\
Quercetina & 5.87 & 7.32 \\
Camferol & 5.97 & 7.42 \\
Crisina & 6.37 & 7.95 \\
\hline
\end{tabular}

Nota: Valores reportados en eV.

Elaboración propia.

Por otro lado, es curioso ver cómo los alcaloides presentan valores, incluso ligeramente más pequeños que los flavonoides, de donde surge una conclusión importante: que los alcaloides son ligeramente más susceptibles a la donación de electrones, con una diferencia de $0.23 \mathrm{eV}$ con respecto a la quercetina, correspondiente $a \sim 5 \mathrm{kcal} / \mathrm{mol}$.

Del lado del mapeo de los orbitales moleculares, se puede observar de la figura 3 (d), que la distribución del HOMO en la quercetina se encuentra en la fracción catecol principalmente, dicha porción de la molécula es la principal aportadora al momento de donar un electrón. Para el camferol (figura 3 [e]) la distribución de este orbital se da entre el grupo fenólico y una muy pequeña contribución del resorcinol. Sin embargo, para la crisina (figura 3 [f]), el HOMO está totalmente situado en el anillo de resorcinol. Esto tiene sentido ya que su potencial de ionización es mayor en $0.6 \mathrm{eV}$ frente a la quercetina, y se ha reportado que los compuestos con resorcinol son menos activos (Ortega-Moo et al., 2016, p. 177).

Para los alcaloides es curioso observar que el orbital HOMO no se encuentra situado en la fracción fenólica como se esperaría. Para los tres alcaloides,

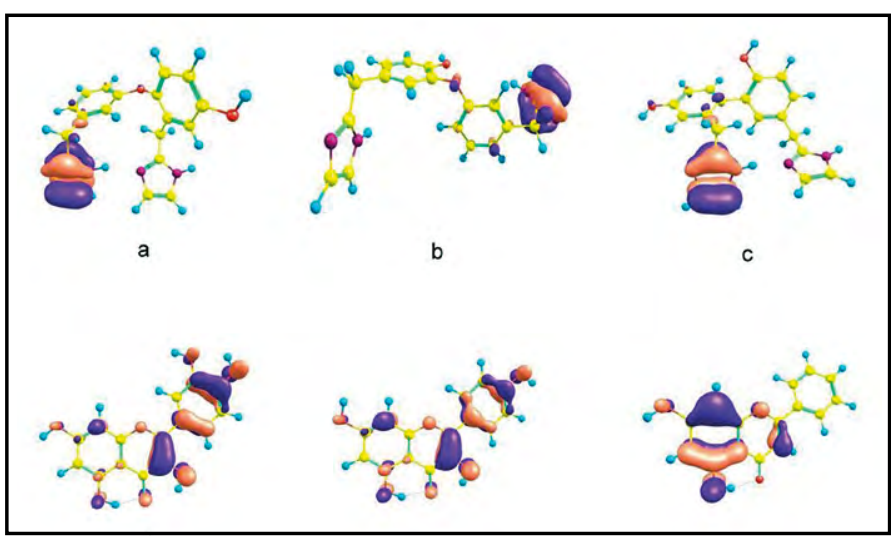

Figura 3. Orbital HOMO obtenido con B3LYP/6-31+G(d) para: a) Lepidina D, b) Lepidina E, c) Lepidina F, d) quercetina, e) camferol y f) crisina. Valores de la isosuperficie de 0.05.

Elaboración propia.

el HOMO se encuentra totalmente localizado en el anillo de imidazol, sin que haya contribución del grupo fenólico sobre este orbital. Por tanto, cualquiera de las lepidinas estudiadas en este trabajo van a ser susceptibles a donar un electrón de la región imidazol y no de la fracción fenol.

Debido a que en un mecanismo SET se da la transferencia de electrones, también es importante ver los sitios donde se puede dar una transferencia de carga por fracciones y no sólo por un electrón completo. Para esto, se evaluó la función de Fukui $\left(f^{-}\right)$, donde se ve un pequeño contraste con lo encontrado en el mapeo del orbital HOMO. Las funciones de Fukui están basadas en la teoría de orbitales frontera, que asocian a los orbitales de valencia la reactividad de cualquier sistema químico. En este caso, la función de Fukui va a predecir los sitios donde es más factible tener un ataque electrofílico $\left(f^{-}\right)$, lo que se espera de un buen antioxidante.

De la figura 4 (d, e) podemos rescatar que para la quercetina y el camferol los sitios susceptibles a una donación de carga se encuentran sobre los carbonos que unen a los grupos catecol y fenol, respectivamente. También se observa que se puede dar dicha transferencia de carga sobre los grupos - $\mathrm{OH}$ de las fracciones mencionadas anteriormente; mientras que para la crisina (figura 4 [f]), se sigue viendo el mismo comportamiento que el mostrado por el orbital HOMO, ya que se encuentra la $f^{-}$sobre la fracción resorcinol. De la misma manera y con el mismo comportamiento, para la lepidina D, E y F la $f$ - se encuentra situada en el grupo imidazol, con una pequeña contribución en el grupo fenólico en esta última. 
InVESTIGACIÓn Y GIEnCIA DE LA UNIVERSIDAD AUTÓNOMA actividad antioxidante, al menos la Lepidina $\mathrm{F}$ puede fungir como un buen antioxidante e incluso mejor que cualquiera de los flavonoides aquí mostrados. Lo más interesante de este resultado es que el sitio de donde se donaría el electrón es de la fracción imidazol y no del fenol. Según el conocimiento de los autores del presente trabajo esto es totalmente nuevo, ya que no se ha reportado a nivel teórico que un alcaloide imidazólico tenga gran capacidad antioxidante debido a que en su estructura haya un grupo imidazol, en donde éste es el responsable de la donación del electrón en un mecanismo SET.

\section{CONCLUSIONES}

Figura 4. Función de Fukui ( $f-$ ) para: a) Lepidina $D$, b) Lepidina $E$ c) Lepidina F, d) quercetina, e) camferol y f) crisina. Valores de la isosuperficie de 0.005 .

Elaboración propia.

\section{DISCUSIÓN}

Del análisis conformacional podemos decir que los parámetros geométricos en las estructuras estudiadas no varían entre ellas, analizando primordialmente la fracción fenólica para los flavonoides y el imidazol en los alcaloides. En un estudio teórico realizado por Fabijanić, Brala y Pilepić (2015), donde se explora la reactividad química de $\alpha$-tocoferol o vitamina $\mathrm{E}$, el cual es considerado un buen antioxidante, se reporta un valor de $\mathrm{Pl}$ de $8.39 \mathrm{eV}$, lo que sugiere que los flavonoides $y$, por ende, los alcaloides estudiados en este trabajo, son buenos antioxidantes, ya que difieren por debajo del valor reportado en $0.98 \pm 0.44$ $\mathrm{eV}$ respecto al $\alpha$-tocoferol. Es claro que de los valores obtenidos para los alcaloides y flavonoides, los alcaloides tienen un menor potencial de ionización, lo que sugiere que si el $\mathrm{Pl}$ es proporcional a la
En el presente estudio se analizaron tres alcaloides y tres flavonoides en el marco de la teoría de funcionales de la densidad. Se determinó su potencial de ionización y se encontró que para el caso de los flavonoides la quercetina es la más activa y en los alcaloides la Lepidina $F$ es la que tiene el valor más pequeño, lo que la hace la más susceptible a donar un electrón. Una conclusión importante encontrada en este trabajo es la región donde se encuentra el orbital HOMO, ya que para los alcaloides está situado en la fracción imidazol y no en el fenol, como se esperaba. De los resultados obtenidos en el presente estudio, podemos concluir que los alcaloides podrían fungir como buenos antioxidantes, incluso por encima de los flavonoides. Como perspectiva, es necesario llevar a cabo una investigación más robusta para ratificar la hipótesis del presente trabajo, y verificar si realmente los alcaloides con fracción imidazol pueden tener gran capacidad antioxidante, y abrir camino al estudio de nuevos compuestos que contengan esta fracción. 
- Aksu, K., Topal, F., Gulcin, I., Tümer, F., \& Göksu, F. (2015). Acetylcholinesterase inhibitory and antioxidant activities of novel symmetric sulfamides derived from phenethylamines. Archiv der Pharmazie: Chemistry in Life Sciences, 348(6), 446455.

- Becke, A. D. (1993). Density-functional thermochemistry. III. The role of exact exchange. The Journal of Chemical Physics, 98(7), 5648-5652. doi: 10.1063/1.464913

- Ditchfield, R., Hehre, W. J., \& Pople, J. A. (1971). Self-consistent molecular-orbital methods. IX. An extended gaussian-type basis for molecular-orbital studies of organic molecules. The Journal of Chemical Physics, 54 (2), 724-728. doi: 10.1063/1.1674902

- Fabijanić, I., Brala, C. J., \& Pilepić, V. (2015). The DFT local reactivity descriptors of $\alpha$-tocopherol. Journal of Molecular Modeling, 21 (4), 99. doi: 10.1007/s00894-015-2644-y

- García-Hernández, E., \& Garza, J. (2017). Reactivity sites in dopamine depend on its intramolecular hydrogen bond. Journal of the Mexican Chemical Society, 61 (3), 222-228.

- Gülcin, I., Büyükokuroglu, M. E., \& Küfrevioglu, O. I. (2003). Metal chelating and hydrogen peroxide scavenging effects of melatonin. Journal of Pineal Research, 34 (4), 278-281.

- Gülcin, I., Büyükokuroglu, M. E., Oktay, M., \& Küfrevioglu, O. I. (2003). Antioxidant and analgesic activities of turpentine of Pinus nigra Arn. subsp. pallsiana (Lamb.) Holmboe. Journal of Ethnopharmacolology, 86(1), 51-58.

- Halake, K., Birajdar, M., \& Lee, J. (2016). Structural implications of polyphenolic antioxidants. Journal of Industrial and Engineering Chemistry, 35, 1-7. doi: 10.1016/j.jiec.2016.01.003

- Havsteen, B. H. (2002). The biochemistry and medical significance of the flavonoids. Pharmacology \& Therapeutics, 96(2-3), 67-202.

- Maier, U. H., Gundlanch, H., \& Zenk, M. H. (1998). Seven imidazole alkaloids from Lepidium sativum. Phytochemistry, 49(6), 1791-1795.

- Middleton, E., Kandaswami, C., \& Theoharides, T. C. (2000). The effects of plant flavonoids on mammalian cells: Implications for inflammation, heart disease, and cancer. Pharmacological Reviews, 52(4), 673-751.
- Ortega-Moo, C., Garza, J., \& Vargas, R. (2016). The substituent effect on the antioxidant capacity of cathecols and resorcinols. Theoretical Chemistry Accounts, 135, 177-188. doi: 10.1007/s00214-016-1932-7

- Parr, R. G., \& Yang, W. (1984). Density functional approach to the frontier-electron theory of chemical reactivity. Journal of the American Chemical Society, 106(14), 4049-4050. doi: $10.1021 / \mathrm{ja00326a036}$

- Qian, J. Z., Wang, B. C., Fan, Y., Tan, J., \& Yang, X. (2015). QSAR study of flavonoid-metal complexes and their anticancer activities. Journal of Structural Chemistry, 56(2), 338-345. doi: $10.1134 /$ S0022476615020195

- Sepúlveda-Jiménez, G., Porta-Ducoing, H., \& Rocha-Sosa, M. (2003). La participación de los metabolitos secundarios en la defensa de las plantas. Revista Mexicana de Fitopatología, 21 (3), 355-363.

- Shahidi, F., \& Zhong, Y. (2010). Lipid oxidation and improving the oxidative stability. Chemical Society Reviews, 39(11), 40674079. doi: 10.1039/b922183m

- Srivastava, A., Tandon, P., Ayala, A. P., \& Jain, S. (2011). Solid state characterization of an antioxidant alkaloid boldine using vibrational spectroscopy and quantum chemical calculations. Vibrational Spectroscopy, 56(1), 82-88. doi: 10.1016/j. vibspec.2010.08.001

- Valiev, M., Bylaska, E. J., Govind, N., Kowalski, K., Straatsma, T. P., Van Dam, H. J. J., ..., de Jong, W. A. (2010). NWChem: a comprehensive and scalable open-source solution for large scale molecular simulations. Computer Physics Communications, 181(9), 1477-1489. doi: 10.1016/j. cpc.2010.04.018

- Villuendas-Rey, Y., Alvarez-ldaboy, J. R., \& Galano, A. (2015). Assessing the protective activity of a recently discovered phenolic compound against oxidative stress using computational chemistry. Journal of Chemical Information and Modeling, 55(12), 2552-2561. doi: 10.1021/acs.jcim.5b00513

- Zou, L., \& Akoh, C. C. (2015). Antioxidant activities of annatto and palm tocotrienol-rich fractions in fish oil and structured lipid-based infant formula emulsion. Food Chemistry, 168, 504511. doi: 10.1016/j.foodchem.2014.07.098 